\title{
Evaluating IP, MPLS and MPLS RSVP-TE Networks
}

\author{
Oyeleke G. Akinsipe \\ Ulcanado Communications Ltd \\ Abuja, Nigeria
}

\author{
Oluwatosin O. Akinsipe \\ Ulcanado Communications Ltd \\ Abuja, Nigeria
}

\begin{abstract}
The basic components of a network can be used in its enhancements through the provision of some reliable services. In order to achieve these provisions of reliable services, various technologies have been put in place. One of these technologies is MPLS and it provides Quality of Service and this is done by delivering such services with high transmission speed, low delays and low losses. Due to the ability to implement Traffic Engineering in MPLS, it can be used to efficiently implement real-time applications. Signaling protocols such as RSVP-TE and CR-LDP are used for Traffic Engineering in MPLS.

In this paper, the modeling of IP, MPLS and MPLS RSVP-TE (with path reserved for voice traffic) networks are presented and the performance parameters of the networks are compared. OPNET modeler 16.0 is used to simulate all the networks and the comparison is made for parameters such as Voice packet delay variation, Video packet end-to-end delay variation and voice packet reception rate.
\end{abstract}

\section{General Terms}

IP, MPLS, Real-time Applications, Traffic Engineering, Networks

\section{Keywords}

IP, MPLS, RSVP-TE, TE, Traffic Engineering, OPNET, Simulator, Ulcanado

\section{INTRODUCTION}

Since the introduction of the world wide web, there has been a tremendous growth of the internet from just been a network of moderate proportional use, which was basically used by the academic community as well as for research and has now become a very large public data network, which plays a major role in the lives of people due to the large range of services and applications it offers and delivers. The high increase in the number of internet users made services such as telephone and television to reach their customers via the internet and this has been forcing Internet Service Providers (ISPs) to improve their quality of service. With this increase as well as the advances made in real-time applications (voice and video), the traditional routers have the challenges of providing the required high bandwidth, fast routing as well as quality of service support. Due to the challenges of traditional routers to provide these requirements especially for voice and video, methods such as the use of Multi-Protocol Label Switching (MPLS) and so on are now used. Multi-Protocol Label Switching (MPLS) is a fast growing technology, which plays a vital role in providing quality of service $(\mathrm{QoS})$ and traffic engineering. It uses information contained in the labels, which are attached to Internet Protocol (IP) packets to improve the fast forwarding of these packets. MPLS provides scalability as well as congestion control in order to overcome limitations such as high packet loss and excessive delays in the network.

Various researches [1-4] have been carried out on the comparison of IP and MPLS networks.

\section{BACKGROUND}

\subsection{Traditional IP Routing}

In the traditional IP routing, routing tables are built by every router in the network by the use of different routing protocols such as OSPF (Open Shortest Path First), RIP (Routing Information Protocol), IS-IS (Intermediate System-toIntermediate System) or BGP (Border Gateway Protocol). Every router in the network has to individually make routing decisions for each incoming IP packet after the routing tables are built and this is time consuming. A router checks its routing table at the arrival of any packet to verify the next hop for the packet based on the destination address of the packet shown in the IP header of the packet.

\subsection{MPLS}

MPLS (Multi-protocol Label Switching) is an advancing technology, which is mainly responsible for high performance packet control and mechanism [5]. It does this by the information contained in the labels attached to the IP packets to forward such packets through a network. It merges the strength of layer 2 switching and layer 3 routing to form an IP network with a high level of performance. MPLS has evolved into a vital technology which efficiently operates and manages IP networks because of its superior characteristics [6]. The purpose of MPLS is to guarantee speed, traffic engineering, Quality of Service (QoS) and scalability of the network and is also useful for VPNs (Virtual Private Networks). MPLS is not a substitute for IP, but it extends the IP architecture by adding new functions to it. The MPLS domain has two major kinds of switches namely; the MPLS edge switches, which basically consist of the LERs (Label Edge routers) and the MPLS core switches, which basically consist of the LSRs (Label Switch Routers). When a packet enters into an MPLS domain, a label is attached to the packet. A label has no internal structure and is a short and fixed unit. This MPLS label is between the Data link layer and the Network layer and the packets are forwarded based on the MPLS labels.

\subsection{Traffic Engineering}

Traffic Engineering (TE) is a mechanism put in place to control the flow of traffic in networks and it provides the performance optimization of the network resources. The main characteristics of TE are fault-tolerance, optimum resource utilization and resource reservation [7]. The basic objective of the consideration of TE is to improve quality of service of some applications and use the available network resources efficiently. There are some important factors, which are needed for TE. These factors are; Path Selection, Traffic 
Management, Direction of Traffic along Computed Paths and Distribution of Topology Information.

The LSPs in the MPLS network are established and the labels are distributed on each of the hops along the LSPs before packets could be forwarded. The LSPs can be established either by explicitly routed LSP or control driven LSP. Control driven LSPs can also be referred to as hop-by-hop LSP and are set by the use of LDP protocol. Explicitly routed LSPs can also be referred to as constraint based LSPS (CR-LSPs), which are specified in the setup message. At each hop, a label request is sent to the next hop along the LSP [8].

\section{METHODOLOGY}

OPNET simulator was used to compare the three routing protocols. OPNET is a real-time simulator designed mainly for the design and analysis of network models [9]. The IP network topology, the MPLS network topology and the MPLS RSVP-TE network topology used to carry out this research consist of 54 routers each as shown in Figure 1, Figure 2 and Figure 3 respectively.

For the MPLS RSVP-TE network, path was reserved for voice traffic from the West_Router to the East_Router along path $\mathrm{R} 1$ to $\mathrm{R} 13$.

Each network was designed in such a way that there were five (5) different types of applications, which are FTP, HTTP, Email, Voice and Video applications transmitted within the network.

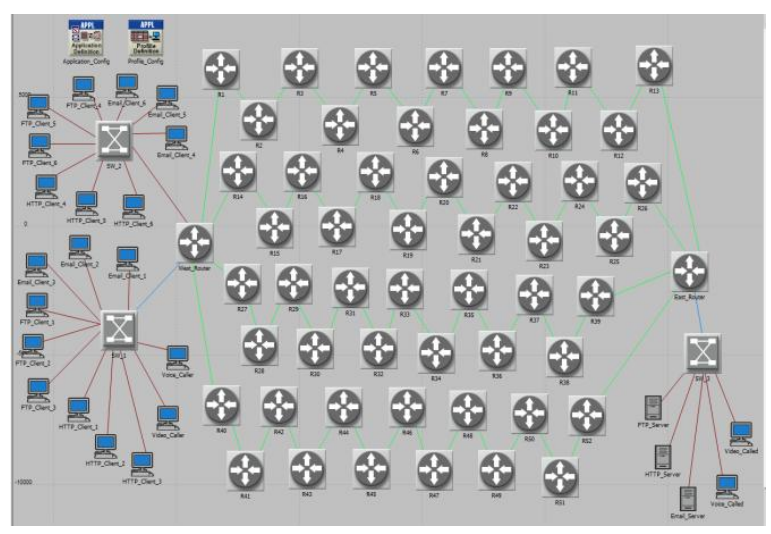

Figure 1: The IP Network Topology

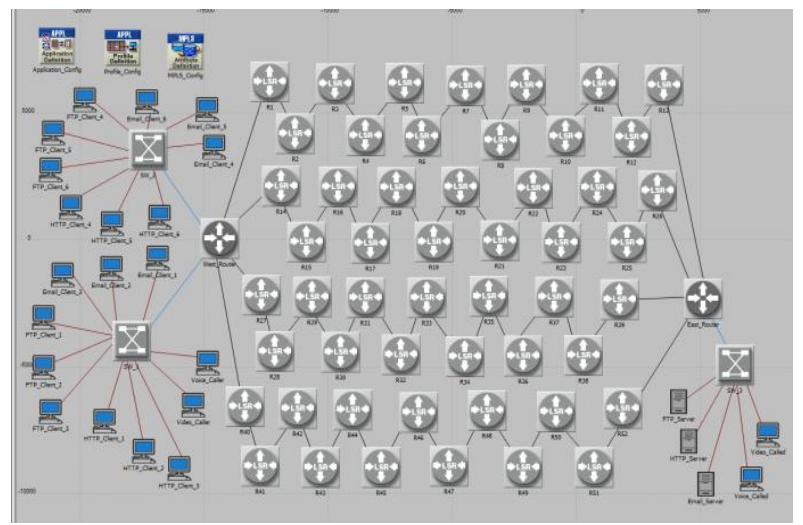

Figure 2: The MPLS Network Topology

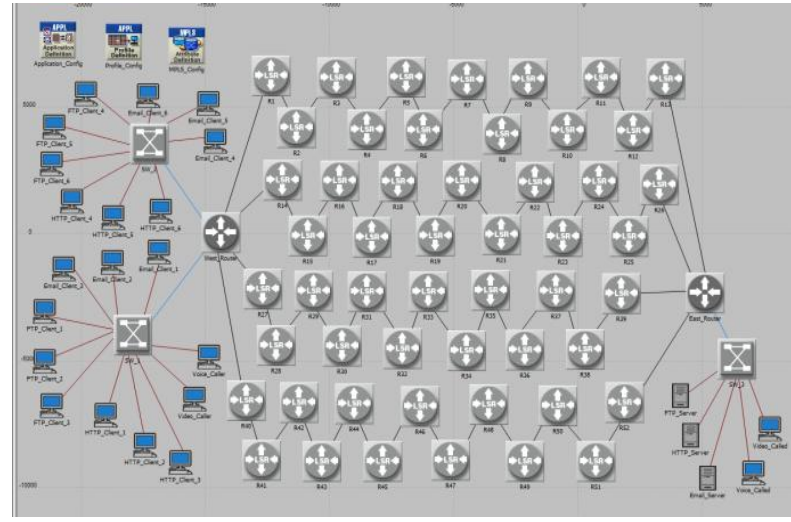

Figure 3: The MPLS RSVP-TE Network Topology

\section{RESULTS AND ANALYSIS}

\subsection{Voice Packet Delay Variation}

From Figure 4, it can be seen that the voice packet delay variation for the traditional IP network increased rapidly throughout the duration of the simulation. It has the highest voice packet delay variation of about $2.43 \mathrm{secs}$ at the end of the simulation and this was due to other traffic in the network. The other traffic such as video, HTTP, FTP and Email were responsible for this delay as the network was congested by the traffic.

Also, from Figure 4, the voice packet delay variation for the MPLS network increased rapidly throughout the simulation period. It has its highest voice packet delay variation of $2.55 \mathrm{secs}$ at the end of the simulation and this was due to the presence of other traffic such as FTP, video, HTTP and Email in the network, which congested the network.

Moreover, from Figure 4, the voice packet delay variation for the MPLS RSVP-TE network increased slightly throughout the simulation period. It has its highest voice packet delay variation of about $0.68 \mathrm{sec}$. It has a very low voice packet delay variation, because of the reserved path for voice traffic.

Also, the traditional IP network has the highest voice packet delay variation for most part of the simulation compared to those of the MPLS and the MPLS RSVP-TE networks, due to the experience of congestion of traffic for most part of the simulation period, but at the end of the simulation, the voice packet delay variation was the highest for MPLS network compared to the traditional IP and MPLS RSVP-TE networks.

The voice packet delay variation for the MPLS network is $0.12 \mathrm{sec}$ higher than the voice packet delay variation for the traditional IP network and 1.87secs higher than the voice packet delay variation for the MPLS RSVP-TE network while the voice packet delay variation for the traditional IP network is $1.75 \mathrm{secs}$ higher than the voice packet delay variation for the MPLS RSVP-TE network. The MPLS RSVP-TE has the lowest voice packet delay variation due to the reserved path for the transmission of voice traffic from the source to the destination. 


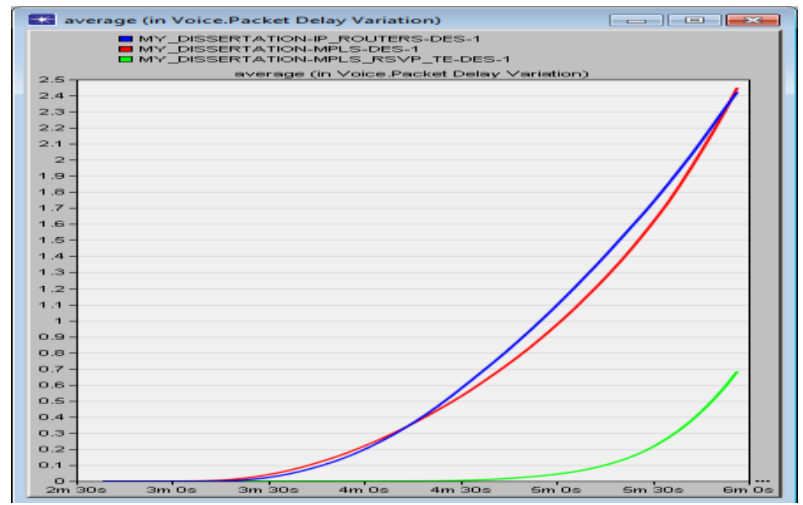

Figure 4: The Voice Packet Delay Variation

\subsection{Voice Traffic Sent and Received}

For the traditional IP network, it can be seen from Figure 5 that the voice traffic sent increased rapidly from 0byte/sec at about 160secs after the start of the simulation to about $109,000 \mathrm{bytes} / \mathrm{sec}$ at the end of the simulation period while from Figure 6, the voice traffic received for the traditional IP network increased rapidly from 0byte/sec at about 160secs after the start of the simulation to about $98,000 \mathrm{bytes} / \mathrm{sec}$ at the end of the simulation period. The voice traffic sent was about $109,000 \mathrm{bytes} / \mathrm{sec}$ at the end of the simulation. The voice traffic received was less than that sent due to congestion of the network other traffic within the network and this led to some of the voice traffic being dropped. Since UDP is the transport layer protocol, there is no retransmission of voice traffic between the voice clients (voice_caller and voice_called).

Also, it can be seen that the voice traffic sent in the MPLS network increased rapidly from 0 byte/sec at about 160 secs after the start of the simulation to about 83,500bytes/sec at the end of the simulation period while the voice traffic received in the MPLS network increased rapidly from 0byte/sec at about 160secs after the start of the simulation to about $51,500 \mathrm{bytes} / \mathrm{sec}$ at the end of the simulation period. The voice traffic sent was about 83,500bytes/sec at the end of the simulation. The voice traffic received was less than that sent due to congestion of the network by other traffic within the network and this led to some of the voice traffic being dropped.

Furthermore, the voice traffic sent in the MPLS RSVP-TE network increased rapidly from 0byte/sec at about 160secs after the start of the simulation to about $82,000 \mathrm{bytes} / \mathrm{sec}$ at the end of the simulation period while the voice traffic received in the MPLS RSVP-TE network increased rapidly from 0byte/sec at about 160 secs after the start of the simulation to about $80,000 \mathrm{bytes} / \mathrm{sec}$ at the end of the simulation. The voice traffic sent was about 82,000 bytes/sec at the end of the simulation. The voice traffic received was less than that sent due to congestion of the network at switch (SW1).

It can therefore be seen that the dropped voice traffic in the traditional IP network is about $11,000 \mathrm{bytes} / \mathrm{sec}$, which is about $10.09 \%$ of the sent voice traffic. The dropped voice traffic in the MPLS network is about 32,000bytes/sec, which is about $38.32 \%$ of the sent voice traffic. The dropped voice traffic in the MPLS RSVP-TE network is about $2,000 \mathrm{bytes} / \mathrm{sec}$, which is about $2.44 \%$ of the sent voice traffic. The voice traffic reception rate for the MPLS RSVP-TE network is higher than those for the traditional IP network and the MPLS network, while that for IP network is higher than that for the MPLS network. The MPLS RSVP-TE network has a higher voice traffic reception rate than the traditional IP network and the MPLS network, because there is reservation of path for the voice traffic in the MPLS RSVP-TE network.

It can therefore be concluded that in terms of the voice traffic, the MPLS RSVP-TE network performs better than both the MPLS network and the traditional IP network.

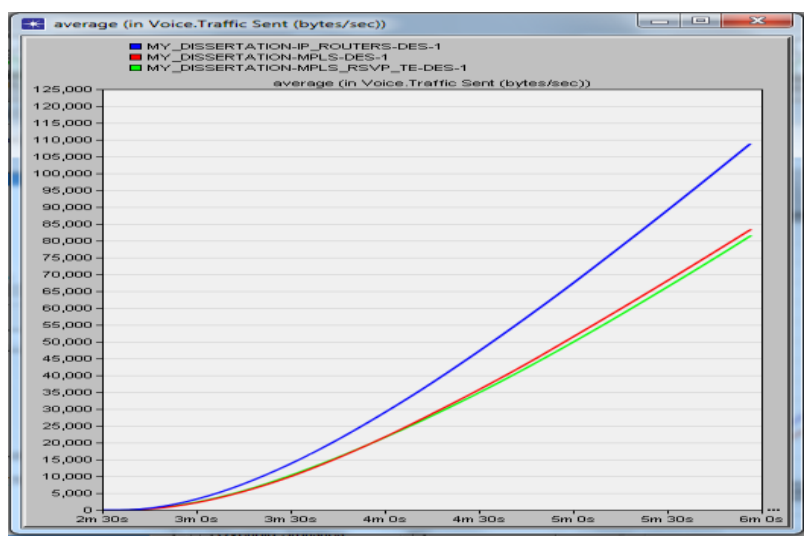

Figure 5: The Voice Traffic Sent

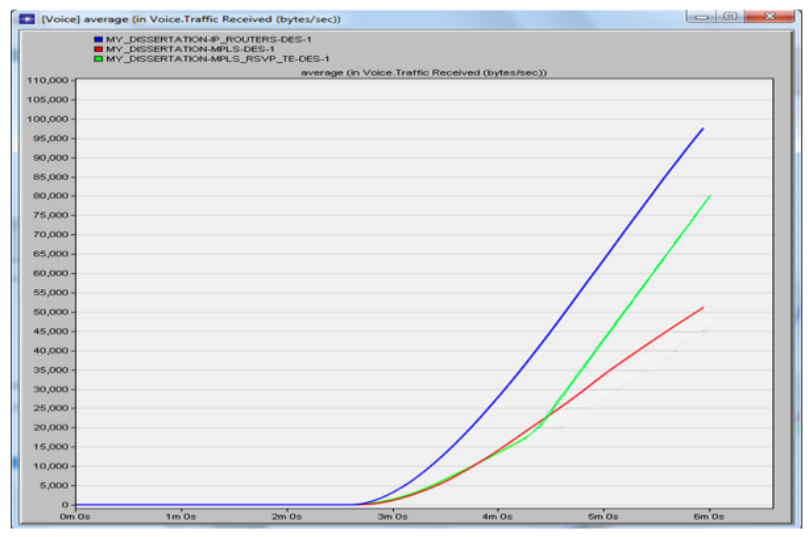

Figure 6: The Voice Traffic Received

Table 1: Voice Traffic Details for IP, MPLS and MPLS RSVP-TE Networks

\begin{tabular}{|c|c|c|c|c|}
\hline Network & $\begin{array}{c}\text { Voice } \\
\text { Traffic } \\
\text { Sent } \\
\text { (byte/sec) }\end{array}$ & $\begin{array}{c}\text { Voice } \\
\text { Traffic } \\
\text { Received } \\
\text { (byte/sec) }\end{array}$ & $\begin{array}{c}\text { Voice } \\
\text { Traffic } \\
\text { Dropped } \\
\text { (byte/sec) }\end{array}$ & $\begin{array}{c}\text { Voice } \\
\text { Traffic } \\
\text { Dropped } \\
(\%)\end{array}$ \\
\hline IP & 109,000 & 98,000 & 11,000 & 10.09 \\
\hline MPLS & 83,500 & 51,500 & 32,000 & 38.32 \\
\hline MPLS & 82,000 & 80,000 & 2,000 & 2.44 \\
RSVP-TE & \multicolumn{2}{|c|}{} \\
\hline
\end{tabular}

\subsection{Video Packet End-to-End Delay}

From Figure 7, it can be seen that the end-to-end video packet delay for the traditional IP network started to increase from 0 sec at 3 mins after the start of the simulation to about $37.5 \mathrm{secs}$ at the end of the simulation. This increase was rapid.

Furthermore, the end-to-end video packet delay for the MPLS network was 0sec till after the first 3 mins of the simulation, where it started to rapidly increase from 0 sec to about $20 \mathrm{secs}$ at the end of the simulation period. Also, it can be seen for the MPLS RSVP-TE network that the end-to-end video packet 
delay was 0sec till after the first 3 mins of the simulation period, where it started to rapidly increase from 0sec to about $27 \mathrm{secs}$ at the end of the simulation.

It can be seen that the video packet end-to-end delay for the traditional IP network is higher than that for the MPLS network as well as that for the MPLS RSVP-TE network. This is so because there are more queues in the traditional IP network and the video traffic contends for bandwidth along with other traffic in the network. The MPLS RSVP-TE network has a higher video packet end-to-end delay than the MPLS network. This is due to the reserved path for voice traffic in the MPLS RSVP-TE network and this makes the video traffic and other traffic contend for the other paths in the network. This causes more queues in the network.

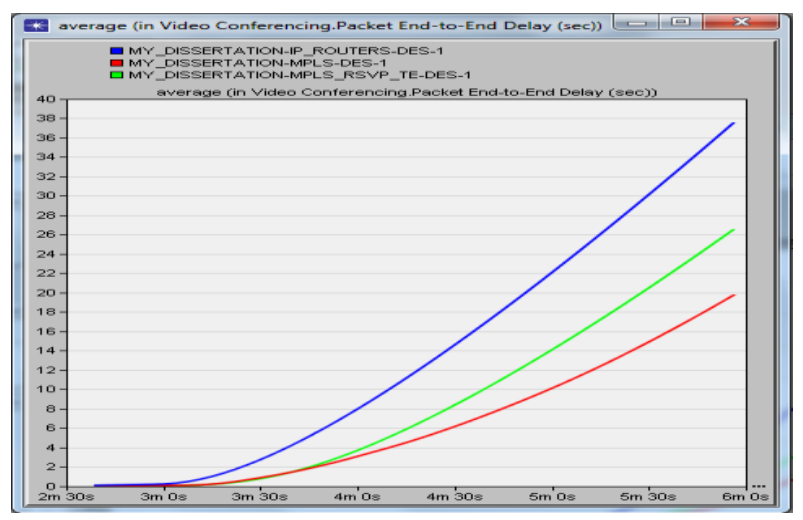

Fig 7: The Video Packet End-to-End Delay

\section{CONCLUSION}

After critically analyzing the results, it can be said that the IP network has the highest Voice Packet Delay Variation (higher than those of the MPLS and MPLS RSVP-TE networks) and the MPLS network has a higher Voice Packet Delay Variation than the MPLS RSVP-TE network. This implies that the MPLS RSVP-TE network experienced the lowest Voice Packet Delay Variation.

Furthermore, it can be said that the dropped voice traffic in the traditional IP network is about $10.09 \%$ of the sent voice traffic while that in the MPLS network is about $38.32 \%$ of the sent voice traffic and that in the MPLS RSVP-TE network is about $2.44 \%$ of the sent voice traffic.

It can also be said that the IP network has the highest Video Packet End-to-End Delay (higher than those of the MPLS and MPLS RSVP-TE networks) and the MPLS RSVP-TE network has a higher Video Packet End-to-End Delay than the MPLS network. This implies that the MPLS network experienced the lowest Video Packet End-to-End Delay.

Therefore, it can be concluded that the MPLS network performs better than both the IP and MPLS RSVP-TE networks in terms of Video Packet End-to-End Delay while the MPLS RSVP-TE network has the best performance (better than those of the MPLS network and the IP network) for voice traffic due to the reserved path and the MPLS network has a better performance for voice traffic than the IP network.

\section{ACKNOWLEDGEMENTS}

We are grateful to the Almighty God, the Giver of life and our parents and siblings for their support and encouragement. We are also grateful to the board members and staff of Ulcanado Communications Ltd for their immense support towards the completion of this work. We are grateful to Dr Maozhen Li for his support.

\section{REFERENCES}

[1] O. Akinsipe et al. "Comparison of IP, MPLS and MPLS RSVP-TE Networks Using OPNET," International Journal of Computer Applications (IJCA), vol. 58, Nov. 2012, pp. 1-3.

[2] M. A. Rahman et al. "Performance Analysis of MPLS Protocols over Conventional Network," Microwave Conf., China-Japan Joint, Shanghai, Sept. 2008, pp. 763 766.

[3] J. Barakovic et al. "Multimedia Traffic Analysis of MPLS and non-MPLS Network," Multimedia Signal Processing and Communications, 48th Int. Symp. ELMAR-2006, Zadar, Jun. 2006, pp. 285-288.

[4] R. S. Naoum and M. Maswady, "Performance evaluation for VOIP over IP and MPLS," World of Computer Science and Information Technology Journal (WCSIT), vol. 2, pp. 110-114, 2012.

[5] N. F. Mir and A. Chien, "Simulation of Voice over MPLS communications Networks," IEEE ICSS conf., CA, 2002, pp. 389-393.

[6] L. He and P. Botham, "Pure MPLS Technology," in Proc. 3rd International Conf. Availability, Reliability and Security, Barcelona, 2008, pp. 253-259.

[7] X. Xiao et al. "Traffic Engineering with MPLS in the Internet" Global Center Inc. and Michigan State University, USA, vol. 14, pp. 28-33, Mar. 2000.

[8] A. Ghanwani et al. "Traffic Engineering Standards in IP Networks Using MPLS" IEEE Communication Mag. Dec. 1999.

[9] H.M. Asif and M. G. Kaosar, "Performance Comparison of IP, ATM and MPLS Based Network Cores Using OPNET," in 1st IEEE Int. Conf. Industrial and Information Systems, Sri Lanka, Aug. 2006.

[10] C. Metz, "RSVP: general-purpose signaling for IP," Cisco Syst., USA, vol. 3, pp. 95-99, May/Jun. 1999.

[11] M.K. Porwal et al. "Traffic Analysis of MPLS and Non MPLS Network including MPLS Signaling Protocols and Traffic distribution in OSPF and MPLS," Int. Conf. Emerging Trends in Engineering and Technology, ICETET, Jul. 2008.

[12] J. Lawrence, "Designing multiprotocol label switching networks", Communications Magazine, IEEE, vol. 39, pp. 134-142, Jul. 2001

[13] A. Viswanathan, N. Feldman, Z. Wang, R. Callon, Evolution of Multiprotocol Label Switching IEEE Communications Magazine, Vol. 36, Issue 5, pp. 165 173, May. 1998.

[14] D. O. Awduche and B. Jabbori, "Internet traffic engineering using MPLS”, 2002.

[15] A. Guy, "MPLS: Multiprotocol label switching," vol. 48, no.6, pp.20, 2006.

[16] D. E. Comer, Internetworking with TCP/IP: Principles, Protocols and Architectures. 4th Ed., Prentice Hall, pp. 750. 2000. 